\title{
PREVALENCIA DEL VIRUS PAPILOMA HUMANO EN PACIENTES CON DIAGNÓSTICO DE CARCINOMA ESCAMOSO DE LA CAVIDAD ORAL*
}

\author{
Drs. Gina Pennacchiotti ${ }^{1}$, Raúl Sáez ${ }^{1}$, María J. Martínez ${ }^{2}$, \\ Marcela Cárcamo ${ }^{3}$, Rodrigo Montes ${ }^{4}$ \\ 1 Facultad de Odontología, Universidad de Chile. \\ 2 Programa de Virología, Instituto de Ciencias Biomédicas, Facultad de Medicina, Universidad de Chile. \\ 3 Epidemióloga, Registro Hospitalario, Instituto Nacional del Cáncer. \\ 4 Jefe Equipo Cabeza y Cuello, Instituto Nacional del Cáncer. \\ Santiago, Chile.
}

\begin{abstract}
Human papilloma virus prevalence in patients diagnosed with squamous carcinoma oral cavity
\end{abstract}

Aim: To establish the prevalence of HPV in patients with diagnose of Oral Squamous cell carcinoma, treated in the Instituto Nacional del Cáncer in fresh tumoral samples. Methods: Cross-sectional study. The inclusion criteria were patients over 18 years, histological diagnosis of Oral Squamous cell carcinoma, that haven't received any previous cancer treatment, in normal physical and mental conditions. All patients signed an informed consent. Samples consisting of fresh biopsies of lesions with clinical and pathological diagnosis were made. The samples were processed in the Programme of Virology, Faculty of Medicine, Universidad de Chile, through PCR and genotyped by Linear Array® (ROCHE). Results: The prevalence of VPH obtained in this patients was of $9.09 \%(2 / 22)$, corresponding 1 to VPH 56. The positive patients for VPH resulted to be a man of 35 years and a woman of 73 years $(9 \%)$. Conclusion: The prevalence of HPV in this type of patients was $9.09 \%$, similar than the international publications.

Key words: Human papilloma virus, squamous carcinoma, oral cavity.

\section{Resumen}

Objetivos: Determinar la prevalencia del virus papiloma humano (VPH) en pacientes con diagnóstico de carcinoma escamoso de la cavidad oral (COCE), atendidos en el Instituto Nacional del Cáncer (INCANCER). Material y Método: Estudio de corte transversal. Criterios de inclusión: pacientes mayores de 18 años, con diagnóstico histológico de COCE, que no hubiesen recibido ningún tratamiento oncológico previo, en condiciones físicas y mentales normales. Todos los pacientes firmaron el consentimiento informado. Se

\footnotetext{
*Recibido el 23 de julio de 2015 y aceptado para publicación el 21 de septiembre de 2015.

Conflictos de interés: ninguno

Correspondencia: Dra. Gina Pennacchiotti pennacchiotti@gmail.com
} 
les realizó una entrevista y se tomaron muestras que consistieron en biopsias frescas de lesiones con diagnóstico clínico y anatomopatológico de COCE. Las muestras se procesaron en el Programa de Virología de la Facultad de Medicina de la Universidad de Chile, a través de PCR y se genotipificaron mediante Linear Array ${ }^{\circledR}$ (ROCHE). Resultados: La prevalencia de VPH obtenida en estos pacientes fue de un 9,09\% (2/22), correspondiendo 1 a VPH 56. Los pacientes positivos para VPH resultaron ser un hombre de 35 años y una mujer de 73 años (9\%). Conclusión: La prevalencia del VPH en este tipo de pacientes fue de 9,09\%, similar a las publicaciones internacionales.

Palabras clave: Virus papiloma humano, cáncer escamoso, cavidad oral.

\section{Introducción}

La infección por el virus papiloma humano (VPH) es una de las infecciones de transmisión sexual más prevalentes en el mundo. En Estados Unidos, se describen cerca de 5,5 millones de casos nuevos anuales, con una prevalencia estimada de 20 millones y se presume que $20-40 \%$ de las mujeres sexualmente activas, estarían infectadas con $\mathrm{VPH}^{1}$.

Según la literatura, la única localización no genito-anal, en la cual se ha observado una asociación igualmente sólida a favor de un rol etiológico del VPH, corresponde a un grupo de cánceres de cabeza y cuello, entre ellos se encuentra el Carcinoma Oral de Células Escamosas².

La asociación de VPH con COCE ha sido reportada según localización: en la cavidad oral con $12 \%-71 \%$ y laringe $4 \%-24 \%{ }^{3-6}$. Además, se ha encontrado una fuerte asociación de VPH con respecto a la localización: en tumores de orofaringe afecta principalmente, con una mayor positividad para VPH, a amígdala y base de lengua. Se ha descrito que el epitelio escamoso que reviste el "anillo de Waldeyer" podría ser particularmente susceptible a la infección por VPH debido a la facilidad que posee el virus de acceder a las células basales de la mucosa en las criptas de la amígdala ${ }^{7}$.

Chile no cuenta actualmente con registros sobre incidencia ni prevalencia para COCE a nivel nacional. Sin embargo, una reciente investigación de morbilidad por cáncer oral y faríngeo en Chile publicada por Riera y Martínez $^{8}$, determinó que aproximadamente el 1,6\% del total de cánceres que afectaba a la población chilena era cáncer oral. Es preocupante que la morbilidad por este cáncer haya experimentado un aumento progresivo de la tasa en un $79,9 \%$ desde el año 1969 al 2004. De éstos, el COCE es el tumor que con mayor frecuencia afecta al área de cabeza y cuello, representando el 90\% del total de los tumores malignos, siendo lengua, el lugar más afectado por esta enfermedad en la cavidad oral ${ }^{8}$.

Es por esto, que esta investigación quiere establecer la prevalencia del VPH en pacientes con diagnóstico de COCE, atendidos en el Instituto
Nacional del Cáncer, centro público de excelencia para el tratamiento de Cáncer.

\section{Material y Método}

Estudio de corte transversal. La población de estudio fueron pacientes adultos, hombres y mujeres con diagnóstico clínico e histopatológico de COCE, que acudieron al Instituto Nacional del Cáncer para ser tratados. Los criterios de inclusión fueron: ser mayor de 18 años, tener diagnóstico histológico de carcinoma escamoso de cavidad oral, no haber recibido ningún tratamiento oncológico previo local ni sistémico. Los criterios de exclusión fueron: no aceptabilidad por parte del paciente, antecedente de cáncer escamoso de cabeza y cuello previamente tratado en INCANCER u otro centro, paciente tratado con quimioterapia y/o radioterapia previamente en otro centro por un cáncer escamoso de cabeza y cuello y pacientes embarazadas o en período de lactancia.

A cada paciente se le aplicó el Procedimiento de Consentimiento Informado, el cual fue revisado y aprobado por el Comité de Investigación y Ética del Servicio de Salud Metropolitano Norte.

De cada paciente se obtuvo una muestra fresca, de lesiones con diagnóstico clínico y anatomopatológico de COCE. Las biopsias las realizó un cirujano oncólogo del equipo de Cabeza y Cuello del INCANCER durante la cirugía, mediante el uso de bisturí, tomando una zona representativa de más o menos $0,5 \mathrm{~cm} \times 0,5 \mathrm{~cm}$, evitando la zona más necrótica del tumor, las que posteriormente se introdujeron en tubos "screu caps". Las muestras se transportaron inmediatamente, dentro de un contenedor en frío (aproximadamente $8^{\circ} \mathrm{C}$ ) y fueron trasladadas al laboratorio de Virología de la Facultad de Medicina de la Universidad de Chile, donde se almacenaron a $-80{ }^{\circ} \mathrm{C}$ para su posterior procesamiento y análisis.

De cada muestra, se obtuvo el material genético mediante la técnica de extracción de ADN, Proteinasa K (Extracción de ADN (E. Durigon) Brasil) y se cuantificó la concentración de ADN presente en cada muestra mediante espectrofotometría en el equipo Biomate 3, Termo Spectronic. 
Las muestras fueron sometidas a detección de un fragmento del gen de la $\beta$-globina humana mediante PCR convencional, la que permite determinar la calidad del ADN de cada muestra y la presencia de posibles inhibidores de la PCR. Se utilizaron partidores PC03 y PC04 descritos que delimitan una región de 110 pares de base $(\mathrm{pb})$.

\section{Detección de VPH mediante PCR convencional}

Para la detección de VPH, se realizó una PCR convencional con partidores universales GP5+/GP6+ en el equipo Thermal Cycler PXE0, 2 (Thermo Electron USA). En todas las reacciones de amplificación, se incluyó como control positivo, una muestra de DNA purificado de células HeLa, que contienen ADN de VPH-18, y como control negativo agua libre de nucleasas. La amplificación se realizó en el termociclador Thermal Cycler PXE0, 2 (Thermo Electrón USA), bajo las siguientes condiciones: una etapa preliminar de denaturación a $96^{\circ} \mathrm{C}$ por $1 \mathrm{~min}$, ciclos a $96{ }^{\circ} \mathrm{C}$ por $1 \mathrm{~min}, 55^{\circ} \mathrm{C}$ por $1 \mathrm{~min}$, y una etapa final de $72{ }^{\circ} \mathrm{C}$ por $1 \mathrm{~min}$.

\section{Genotipificación de VPH mediante ensayo de Linear Array}

Todas las muestras se genotipificaron utilizando el kit Linear Array (LINEAR ARRAY HPV Genotyping Test, Roche). Este ensayo detecta treinta y siete genotipos de VPH: 6, 11, 16, 18, 26, 31, 33, $35,39,40,42,45,51,52,53,54,55,56,58,59,61$, 62, 64, 66, 67, 70, 71, 72, 73 (MM9), 81, 82 (MM4), 83 (MM7), 84 (MM8), IS39 y CP6108, mediante sondas específicas e incluye un control interno para $\beta$-globina. Este kit incluye cuatro procesos: extracción de ADN de las muestras, amplificación por PCR de VPH y $\beta$-globina, hibridación y detección, que se llevaron a cabo según el fabricante. Se consideraron positivas aquellas tiras en las que apareció una banda azul en alguno de los genotipos. Los resultados obtenidos se interpretaron utilizando la guía de referencia provista por el fabricante.

\section{Análisis estadístico}

Se realizó estadística descriptiva de los datos socio-demográficos para las muestras VPH $(+)$, mediante el uso de tablas. Dichos resultados se entregaron mediante cifras absolutas y relativas. La prevalencia de VPH, se determinó en base al número de casos de VPH divididos por la población.

Cálculo de tamaño de muestra: el cálculo se hizo mediante la fórmula de diferencia de proporciones, con una potencia de $80 \%$, nivel de significación de $5 \%$, obteniéndose un número de 36 pacientes.

El análisis estadístico se realizó mediante el software STATA10.0, utilizando un nivel de confianza de un $95 \%$.

\section{Resultados}

De un total de 22 pacientes que ingresaron en el presente estudio, la edad promedio fue de 53,3 años ( $\mathrm{DE} \pm 14,4)$, la mediana de edad fue de 55,5 años (24-82). El género predominante fue masculino con 12 hombres $(54,5 \%)$ y 10 mujeres $(45,4 \%)$. Todas las muestras analizadas fueron biopsias. La localización más frecuente del COCE, fue el borde izquierdo de la lengua, con un $38,7 \%$ (7), luego borde de lengua derecho, reborde alveolar y piso de boca con un 13,6\% (3). La prevalencia de VPH obtenida en pacientes con COCE fue de un 9,09\% (2/22). Los pacientes positivos para VPH resultaron ser un hombre de 35 años y una mujer de 73 años (9\%). Se observa el contraste entre las características clínicas, epidemiológicas, como también relacionadas con sus hábitos de riesgo (Tabla 1). Se extrajo ADN de las 22 muestras de COCE. Todas obtuvieron concentraciones medibles de DNA mediante espectrofotometría. Las 22 muestras analizadas resultaron positivas para la amplificación del fragmento del gen de $\beta$-globina.

\section{Detección de VPH por PCR convencional}

De las 22 muestras, dos de ellas $(9,09 \%)$ resultaron positivas para los partidores (Figura 1).

\section{Determinación de genotipos de VPH mediante el ensayo de Linear Array}

Se detectó sólo un genotipo $(4,5 \%)$, el cual correspondió a VPH-56 (Figura 2). Todas las muestras analizadas mediante Linear Array obtuvieron una banda visible de $\beta$-globina (control interno del kit), lo que confirma un buen proceso de extracción de ADN de las muestras.

\section{Discusión}

Determinar la prevalencia de la infección por VPH en pacientes con COCE es fundamental, ya que ha sido un tema controversial e incierto a nivel mundial, y en Chile aún no se tienen estudios que permitan conocer si existe presencia del virus en la población, si este tiene o no relación con la génesis del COCE, y si influiría en el pronóstico de los pacientes que padecen esta patología.

En este estudio, sólo fue posible la genotipificación de una de las dos muestras positivas para VPH. La consistencia de las muestras de tejido fresco incluidas en este estudio podría explicar que la técnica de Linear Array no sea la más adecuada para ello. Otra posible causa sería, que el kit comercial no contenga el genotipo presente en la muestra, para lo cual se necesitaría secuenciar el amplificado VPH 
Tabla 1. Caracterización de las muestras positivas para VPH

\begin{tabular}{|lll|}
\hline Variable & Muestra $\mathbf{N}^{\circ} \mathbf{5}$ & Muestra $\mathbf{N}^{\circ} \mathbf{6}$ \\
\hline Género & Femenino & Masculino \\
\hline Ldad & 73 años & 35 años \\
\hline Genotizo & Borde lengua izquierdo & Reborde alveolar \\
\hline Tabaco & No determinado & VPH-56 \\
\hline Alcohol & No & No \\
$1^{\text {a relación }}$ & No & Sí, 100 cc diario \\
\hline$N^{\circ}$ parejas sexuales & 27 años & 17 años \\
\hline Sexo oral & 2 & 10 \\
\hline Sexo anal & No & Sí \\
\hline Uso de Preservativo & No & Sí \\
\hline Marihuana & Nunca & Usualmente \\
HTA & No & No \\
\hline Diabetes Mellitus & Sí & No \\
Grado Diferenciación CEC & Sí & No \\
\hline TNM & Pobremente diferenciado & Pobremente diferenciado \\
\hline Recidiva & T1N0M0 & T4N0M0 \\
Fallecido & Sí & Sí \\
\hline
\end{tabular}

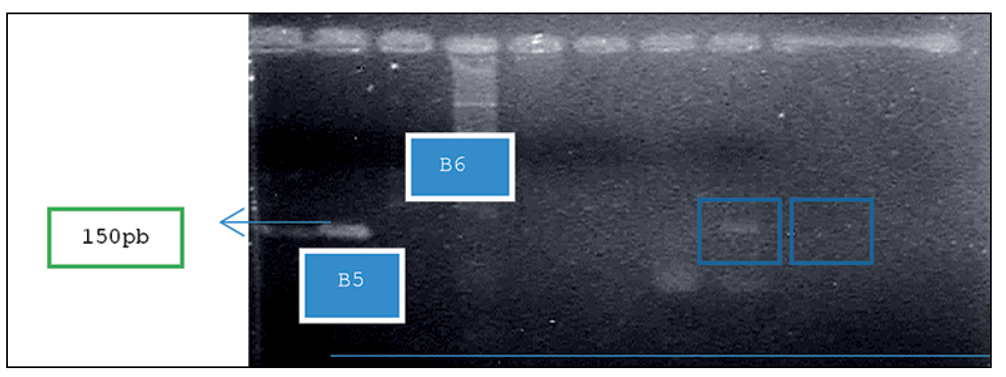

Figura 1. Detección de VPH (150pb) en muestras de COCE en gel de agarosa $2 \%$ teñido con BrEt.

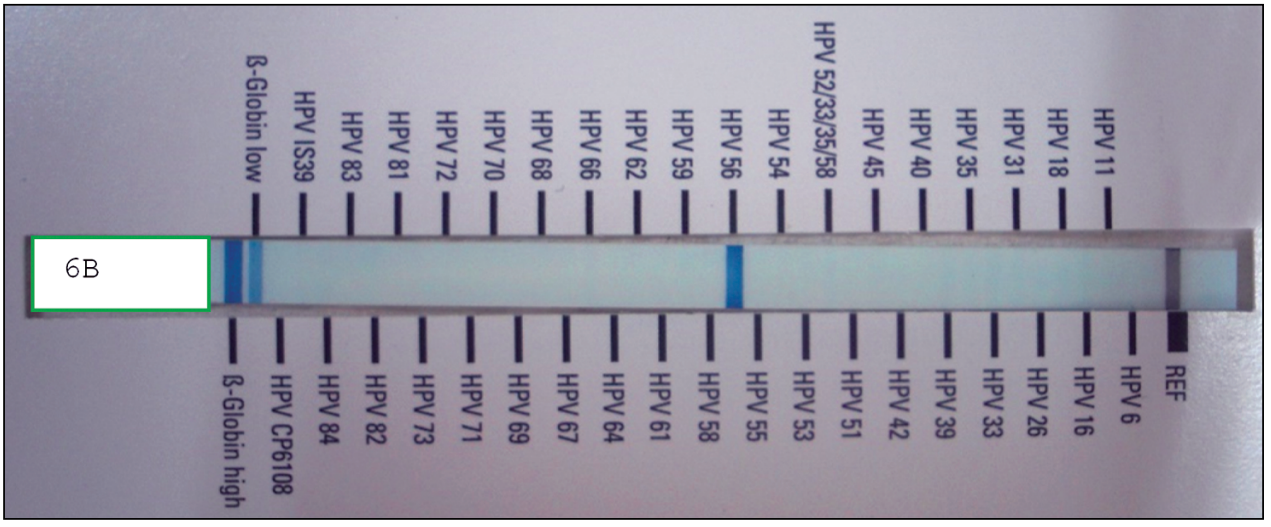

Figura 2. Tiras reactivas obtenidas mediante ensayo de $\mathrm{Li}$ near Array. 
positivo. Otra alternativa sería realizarle un estudio acabado a la muestra que no se pudo genotipificar mediante este ensayo, como una secuenciación, para poder identificar genotipo. Sin embargo, se obtuvo el genotipo 56 en una de las muestras positivas para VPH. VPH-56 es un genotipo de alto riesgo generalmente encontrado en Ca de Cuello Uterino, incluso más frecuente que el 18 , pero no corresponde a los más comúnmente encontrados en cavidad oral, lo que genera la hipótesis de una posible transmisión a través de conductas y hábitos sexuales.

Machado y cols. ${ }^{9}$, realizaron un estudio en 92 pacientes, con la misma metodología que la utilizada en este estudio, reportando que VPH es poco común en carcinomas de cavidad oral $(4 \%)$ y en COCE (6\%). Los resultados de esta investigación son levemente mayores $(9 \%(2 / 22))$, sin embargo, en este estudio no se logró alcanzar el tamaño de muestra. Los tumores VPH positivos se asociaron a pacientes entre 40-60 años y el genotipo 16 fue el más prevalente, pero encontraron otros; $(6,18,33$, $35,45,52,58)$. Este estudio buscó específicamente VPH-16 a través de PCR en tiempo real y genérico para VPH-16, (no se muestran resultados), por ser de alto riesgo, y en la literatura, el más prevalente encontrado en COCE, pero se confirmó su ausencia en la totalidad de las muestras.

El VPH, a diferencia de otros virus, no crece en cultivos celulares convencionales de laboratorio, debido a que tiene tropismo por epitelios y requiere de un epitelio pluriestratificado. Sin embargo, la infección puede ser diagnosticada mediante la presencia de ADN viral utilizando técnicas de hibridación como el Southernblot, dot-blot, hibridación in situ y reacción en cadena de la polimerasa $(\mathrm{PCR})^{10}$. Esta última, permite la detección de VPH con partidores universales y genotipifican mediante partidores específicos para cada genotipo. La detección del genoma viral mediante PCR es el método utilizado en la mayoría de los estudios epidemiológicos ${ }^{11}$. Sin embargo, existen variaciones en la sensibilidad de los diferentes métodos dependiendo del tejido utilizado, tejido en parafina vs tejido fresco, que han dificultado la interpretación y comparación de los reportes existentes.

En el caso de este estudio se utilizaron los partidores GP5+/GP6+ que amplifican un fragmento de $150 \mathrm{pb}$, y se ha reportado que son más sensibles en la detección de ADN de VPH de muestras orales.

El kit de Linear Array, fue creado para la detección de VPH en muestras anogenitales y nuestro estudio se realizó con muestras de tejido fresco de mucosa oral, lo que también podría generar diferencias en la sensibilidad de este ensayo en la detección de VPH aunque se siguieron estrictamente las instrucciones del fabricante.
En relación a los datos sociodemográficos y hábitos de riesgo de la población estudiada; los dos pacientes que arrojaron resultados positivos para la presencia de VPH en sus tumores, un hombre (paciente A) y una mujer (paciente B) muestran datos en relación a la edad (35-73 años respectivamente), donde la literatura hace referencia de que los pacientes VPH positivos son más jóvenes que los VPH negativos, siendo el paciente $\mathrm{A}$, uno de los pacientes más jóvenes del estudio.

La localización (reborde alveolar y borde de lengua respectivamente), no permite concluir nada con respecto a la positividad de VPH en COCE. Aunque diversos estudios han encontrado fuerte asociación del VPH con respecto a la localización; los tumores de orofaringe, sobre todo el de amígdala y base de lengua, presentan una mayor positividad al VPH con respecto a otras, en comparación con localizaciones VPH-negativos ${ }^{7,12}$.

El consumo de alcohol en el paciente hombre positivo para VPH, (100 cc diarios), (destilados fuertes), podría ser un aspecto relevante. Se ha descrito asociación del VPH en pacientes que consumen poco alcohol ${ }^{10}$, sin embargo, es bien conocido que las bebidas alcohólicas contienen etanol, el cual inhibe la producción de la proteína $\mathrm{p} 53^{12}$.

Mucho se ha descrito de la asociación del consumo de tabaco y el COCE, pero también se ha descrito que alrededor de un $25 \%$ de los pacientes diagnosticados con COCE no son fumadores ${ }^{10}$ : pacientes VPH-positivos, requerirían de otros agentes externos para poder desarrollar un COCE (tabaco y alcohol), y que el status fumador actual se relaciona fuertemente con la presencia del VPH y el desarrollo del Cáncer, no así los fumadores antiguos o los no fumadores ${ }^{12}$.

$\mathrm{Al}$ referirnos a las conductas sexuales, se observa que el paciente hombre, tuvo un comienzo de relaciones sexuales a temprana edad (17 años), múltiples parejas sexuales durante su vida, sexo oral y anal utilizando preservativo usualmente. Estos datos concuerdan con el prototipo de individuo que podría presentar VPH como factor de riesgo para COCE, además de su edad ( 35 años) ${ }^{12}$.

En este trabajo, los grados de diferenciación de los COCE positivos para VPH resultaron ser pobremente diferenciados. Gillison encontró una fuerte asociación con respecto a tumores pobremente diferenciados, los cuales fueron más frecuentes en los casos de VPH positivos.

Paz y col. ${ }^{7}$, reportaron que sus pacientes con VPH positivo presentaban mayor estadio en comparación con los VPH negativos, pero no presentó diferencia significativa en la supervivencia a los 3 años de seguimiento. En este estudio la muestra del paciente hombre VPH positivo presentó un estadio avanzado 
en relación al tamaño, pero no se puede concluir nada al respecto.

Actualmente ambos pacientes se encuentran fallecidos y presentaron recidivas de su enfermedad, lo que no coincide con la literatura, que hace referencia a que pacientes con VPH presentan COCE menos agresivos, con una significativa mayor sobrevida libre de enfermedad en comparación con aquellos VPH-negativos, posterior claro, al ajuste por edad, compromiso ganglionar y consumo severo de alcohol. Particularmente el estudio de Gillson, concluyó que existía un $59 \%$ de reducción de fallecer en pacientes con VPH-positivos ${ }^{12}$.

Una de las limitaciones de este estudio fue que no se cumplió con el tamaño de muestra calculado, lo que no permite establecer conclusiones. Sin embargo, aporta la visión de lo que sucede en un centro de referencia nacional. Este tipo de estudios debiesen realizarse con muestras frescas y de manera prospectiva, lo que se dificulta por el tiempo que requiere, mencionando que la captura de pacientes se hace compleja.

Es importante destacar que este estudio se realizó sólo en cavidad oral, ya que la mayoría de los estudios, con mayores tamaños muestrales, incluyen orofaringe, donde existe evidencia que el comportamiento del VPH es diferente.

\section{Referencias}

1. Chuang T, Brashear R. Genital warts. Emedicine. [on line]. 2009. Disponible en: <http://www.emedicine. medscape.com>.

2. Gall S. Female genital warts. Global trends and treatment. Infect Dis Obstet Gynecol. 2001;9:149-54.

3. Ibieta BR, Lizano M, Fras-Mendivil M, Barrera JL, Carrillo A, Ma Ruz-Godoy L, et al. Human Papilloma
Virus Oral Squamous Cell Carcinoma in a Mexican Population. Oral Surg Oral Med Pathol Radiol Endod. 2005;99:311-5.

4. Ortiz M, Torres M, García-Sáiz A. Determinación del virus del papiloma humano (VPH): aspectos técnicos. En: Virus del papiloma humano y cáncer: epidemiología y prevención. ( $4^{\mathrm{a}}$ monografía de la Sociedad Española de Epidemiología). de San José S. García A.M. Madrid: EMISA. 2006. pp. 89-105.

5. Nair S, Pillai MR. Human Papilloma Virus and Disease Mechanism: Relevance to Oral and Cervical Cancers. Oral Dis. 2005;11:350-9.

6. Luo CW, Roan CH, Liu CJ. Human Papilloma Virus in Oral Squamous Cell Carcinoma and Precancerous Lesions detected by PCR-based Gene Chip Array. Int J Oral Maxillof Surg. 2007;36:153-8. Epub 2006 Nov 15.

7. Paz IB, Cook N, Odom-Maryon T, XiE y, Wilczynski SP. Human Papilloma Virus in Head and Neck Cancer. An association of HPV 16 with squamous cell carcinoma of Waldeyer'stonsilar ring. Cancer 1997;9:595-604.

8. Riera P, Martínez B. Morbilidad y Mortalidad por Cáncer Oral y Faríngeo en Chile. Rev Med Chile 2005;133:555-56.

9. Machado J, Reis PP, Zhang T, Simpson C, Xu W, PérezOrdoñez B, et al. Low prevalence of human papilomavirus in oral cavity carcinomas. Head Neck Oncol. 2010;2:6. doi: 10.1186/1758-3284-2-6.

10. Concha R. Marcela, Diagnóstico y terapia del virus papiloma humano, Rev Chil Infect. 2007;24:209-14.

11. Mijares Briñez CM, Suárez R, Castro C, Fuentes SE, Medina C, Pacheco Soler E, y col. Carcinoma de Cavidad Oral y su relación con el Virus Papiloma Humano. Rev Soc Med Quir Hosp Emerg Pérez de León 2007;38:24-33.

12. Gillison ML, Koch WM, Capone RB, Spafford M, Westra WH, Wu L. Evidence for a casual association between human papillomavirus and subset of head and neck cancers. J Natl Cancer Inst. 2009;92:709-20. 